\title{
La evolución de la visión en el diseño y desarrollo de aplicaciones web en proyectos educativos basados en TIC de la ENALLT
}

\author{
Evolution of the vision in the design and development of web applications in educational \\ projects based on TIC of the ENALLT
}

\author{
Sonia Cruz-Techica ${ }^{a}$,Erandeni Maldonado-Figueroa ${ }^{b}$, Alejandro Ortiz-Berrocal ${ }^{c}$, Alejandro \\ Rodríguez-Allende ${ }^{d}$
}

\begin{abstract}
:
At the National School of Languages, Linguistics and Translation of the National Autonomous University of Mexico, the Coordination of Distance Education (CED) develops and promotes educational innovations in blended and e-learning modalities, taking advantage of the benefits of digital technologies to offer quality and relevant educational programs that respond to the necessities and priorities of the different language departments. The CED is formed by multi-disciplinary team integrated for different areas: planning, pedagogy, graphic design and programming engineering, all of them interconnected by a development process that apply in an effective way the technology in learning of languages considering interactions between contents, pedagogics knowledge and technology. Under this scheme, the aim is to ensure that blended and e-learning projects reach the objectives for which they were designed.

The purpose of this paper is to share the experiences and the results this Coordination has acquired from two different standpoints: the web design perspective and the technological point of view. It emphasizes the need of a creative process in order to program the apps that are required in the language learning online courses.
\end{abstract}

\section{Keywords:}

Language learning, visual communication, e-learning and b-learning, graphic interface, Educational development methodology, information and communication technologies

\section{Resumen:}

En la Escuela Nacional de Lenguas, Lingüística y Traducción (ENALLT) de la Universidad Nacional Autónoma de México (UNAM), la Coordinación de Educación a Distancia (CED) desarrolla e impulsa la innovación educativa en la modalidad mixta y a distancia, aprovechando los beneficios de las tecnologías digitales para ofrecer programas educativos pertinentes y de calidad que respondan a las necesidades y prioridades de la entidad. Para ello cuenta con un equipo multidisciplinario integrado por 4 áreas: planeación de proyectos, pedagogía, diseño y comunicación visual, e ingeniería y programación, las cuales están interrelacionadas dentro de un proceso de desarrollo de proyectos que busca aplicar de manera eficaz la tecnología en el aprendizaje de lenguas considerando las interacciones de la tecnología con el conocimiento del contenido de lo que se pretende enseñar y el conocimiento pedagógico para entender las estrategias y metodologías que nos conduzcan al aprendizaje. Bajo este esquema se busca garantizar que los productos realizados cumplan el objetivo para el que fueron diseñados.

Así, el propósito de este trabajo es compartir los resultados obtenidos y la experiencia adquirida a lo largo de 10 años en el desarrollo de proyectos educativos en línea en la ENALLT desde dos puntos de vista, el de diseño web y el tecnológico, destacando el proceso creativo necesario para crear las aplicaciones basadas en TIC aplicadas a la enseñanza y aprendizaje de lenguas extranjeras.

\section{Palabras Clave:}

Aprendizaje de lenguas, comunicación visual, educación a distancia, interfaz gráfica, metodología de desarrollo en proyectos educativos, tecnologías de la información y la comunicación

\footnotetext{
a Correspondence Author, Universidad Nacional Autónoma de México - Escuela Nacional de Lenguas, Lingüística y Traducción, https://orcid.org/0000-0002-2891-5259, Email: sonia@enallt.unam.mx

${ }^{\text {b } U n i v e r s i d a d ~ N a c i o n a l ~ A u t o ́ n o m a ~ d e ~ M e ́ x i c o ~-~ E s c u e l a ~ N a c i o n a l ~ d e ~ L e n g u a s, ~ L i n g u ̈ i ́ s t i c a ~ y ~ T r a d u c c i o ́ n, ~ h t t p s: / / o r c i d . o r g / 0000-0002-9296-~}$ 8275, Email: erandeni@enallt.unam.mx

${ }^{c}$ Universidad Nacional Autónoma de México - Escuela Nacional de Lenguas, Lingüística y Traducción, https://orcid.org/0000-0003-20650046, Email: alex@enallt.unam.mx

d Universidad Nacional Autónoma de México - Escuela Nacional de Lenguas, Lingüística y Traducción, https://orcid.org/0000-0002-13630701, Email: alejandro@enallt.unam.mx
} 


\section{Introducción}

La Coordinación de Educación a Distancia (CED) de la Escuela Nacional de Lenguas Linguística y Traducción (ENALLT) se crea en agosto de 2006 gracias al esfuerzo conjunto del Centro de Enseñanza de Lenguas Extranjeras, ahora ENALLT y la Coordinación de Universidad Abierta y Educación a Distancia (CUAED) con la finalidad de desarrollar y ampliar la oferta educativa a distancia de la ENALLT. La CED se encarga de promover, planificar y dar seguimiento al desarrollo de proyectos de educativos con el uso de las Tecnologías de la Información y la Comunicación (TIC) que respondan a las necesidades actuales de la ENALLT y apoya la formación de profesores de lenguas en aspectos didáctico-pedagógico y tecnológicos para contribuir en su profesionalización docente (Rodríguez, 2016).

La CED cuenta con un proceso de desarrollo formal para fomentar la organización, incrementar la productividad y obtener productos finales de alta calidad que satisfagan las necesidades y expectativas del proyecto. Este proceso de desarrollo está conformado por 3 etapas secuenciales como se muestra en la Figura 1: el pre-desarrollo, el desarrollo y el postdesarrollo.

En el proceso de desarrollo se definen las actividades que los colaboradores de las diferentes áreas han de realizar, el modelo no promueve de ninguna manera trabajos aislados y descontextualizados, por el contrario el proceso es flexible y permite que los colaboradores se involucren en el desarrollo desde las fases iniciales aunque el grueso de su participación se de en fases específicas. Esta flexibilidad de diálogo y comunicación entre los miembros del equipo multidisciplinario está sustentada en el modelo TPACK (Mishra y Koehler, 2006) el cual establece que es necesario interrelacionar, en el contexto educativo, tres tipos de conocimiento: el de contenido, el de la pedagogía y el de la tecnología a fin de integrar de manera eficaz las TIC en la enseñanza de lenguas. La interacción entre estos tres tipos de conocimiento implica tener conciencia sobre cómo estos se influyen entre sí para identificar qué tecnologías son las adecuadas para enseñar y aprender contenidos concretos y cómo influyen para potenciar las estrategias didácticas.

La experiencia de 10 años de desarrollo de proyectos de enseñanza de lenguas con TIC de la CED nos permite por una parte sugerir que la convergencia de los tres tipos de conocimiento implica un entendimiento pertinente de conceptos y principios propios de cada una de las disciplinas involucradas en el desarrollo por parte de cada uno de los colaboradores.

Por otro lado nos permite resaltar la importancia de poner especial atención al público meta al que van dirigidos los proyectos, en nuestro caso, alumnos y profesores de lenguas, para considerar sus características y particularidades para lograr una experiencia de usuario interactiva y funcional. Con ello se pretende que la tecnología sea un medio para que tenga lugar la enseñanza y el aprendizaje y no un obstáculo que genere distracciones o dificultades en el proceso.
En el presente trabajo se expone la evolución experimentada a lo largo de diez años desde la perspectiva del diseño y comunicación visual y la ingeniería y programación, resaltando la influencia que la multidisciplina y el contexto educativo han tenido en dicha evolución. Se tomará como referencia el esquema del proceso de desarrollo para explicar la inmersión de la tecnología en el desarrollo de proyectos educativos en línea.

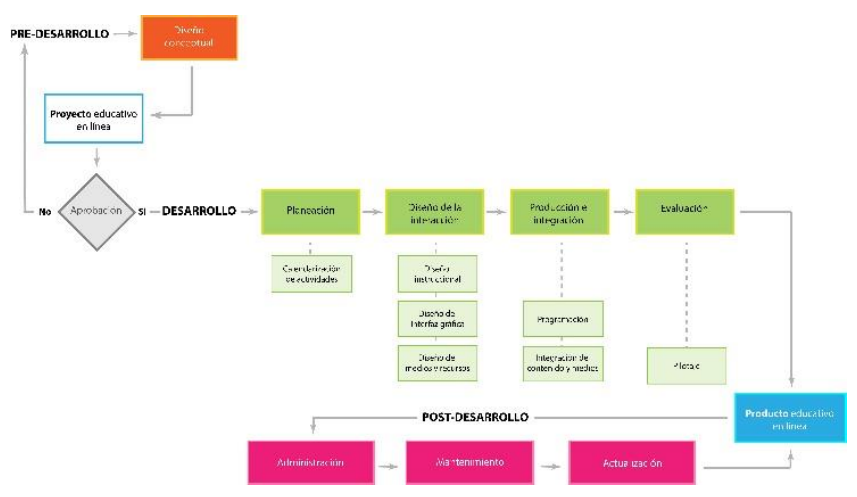

Figura 1: Proceso de desarrollo en la CED

La evolución en el área de diseño gráfico y comunicación visual Badrul H. Khan plasma claramente en su "e-learning framework" ocho dimensiones que hay que tomar en cuenta para planear y diseñar un producto de educación en línea, estas ocho dimensiones deberán centrarse siempre en el estudiante, una de esas dimensiones y sobre la cual versará este apartado de diseño de interfaz.

Para comprender el papel del diseñador y comunicador visual es importante saber qué es una interfaz gráfica, interfaz se define como "el punto de contacto entre los humanos y las máquinas" (Wood, 2015, p.6), si hablamos de interfaz gráfica de usuario (GUI) sumamos una cualidad visual que usa signos gráficos y metáforas visuales y se puede definir como el medio en el cual las personas interactúan con una computadora manipulando directamente los objetos que observan en el monitor.

Con el paso de los años y la adquisición de experiencia, el trabajo de desarrollo de interfaces gráficas en la CED ha ido cambiando y sumando al tomar en cuenta el constante avance tecnológico y las necesidades de los estudiantes y asesores. Cuando la CED inició sus trabajos de desarrollo, el diseño de interfaz gráfica únicamente consideraba el look \& feel del sitio, el día de hoy ha cambiado y se tiene un enfoque más amplio que incluye además la organización de la información, el diseño de la interacción, el diseño textual y la usabilidad, incluir estos aspectos nos da como resultado una experiencia de usuario positiva, es decir una satisfacción global de uso dónde se reduce el esfuerzo cognitivo y los errores al realizar una tarea.

A continuación se explica brevemente cada uno de estos aspectos que hoy se consideran en el trabajo de desarrollo de una interfaz gráfica dentro de la CED:

- La arquitectura de la información es la organización, clasificación y descripción clara del contenido de un 
sitio web, lo que pretende es brindar a las personas los mecanismos necesarios para encontrar lo que buscan.

- Diseño de interacción es el flujo de navegación y la relación entre los elementos que forman parte del sitio, es el comportamiento interactivo de un sitio: el usuario percibe la información, la interpreta y toma decisiones.

- El diseño textual, es la manera en que se presenta el contenido y el estilo tipográfico.

- Usabilidad se define como la rapidez con que se puede aprender a utilizar algo, la eficiencia al utilizarlo, y cuánto le gusta a los usuarios (J. Nielsen, H. Loranger, 2007).

Dicho lo anterior es claro que a lo largo de los años la forma como se ha desarrollado una interfaz gráfica de usuario dentro de la Coordinación ha ido cambiado y se han ido incorporando nuevos elementos; actualmente este trabajo de conceptualización, diseño, construcción e implementación de la interfaz gráfica se puede dividir en cuatro fases que se presentan a continuación.

1a. fase: Organización de la información

Para que un sitio sea eficaz y eficiente la arquitectura de la información debe ser clara. En esta primera fase se planea y trabaja en la arquitectura de la información del entorno virtual de aprendizaje. El uso de diagramas es ideal para poder conceptualizar un sitio, este mapa refleja a primera vista el diseño básico de la estructura y su funcionamiento, así como los contenidos principales como se ilustra en la Figura 2. Como se muestra en la Figura 3 un mapa de sitio también nos ayuda a definir el flujo de navegación, el sitio web presenta diversos caminos, y el estudiante se mueve por estas alternativas que harán que el sitio genere los resultados que busca.

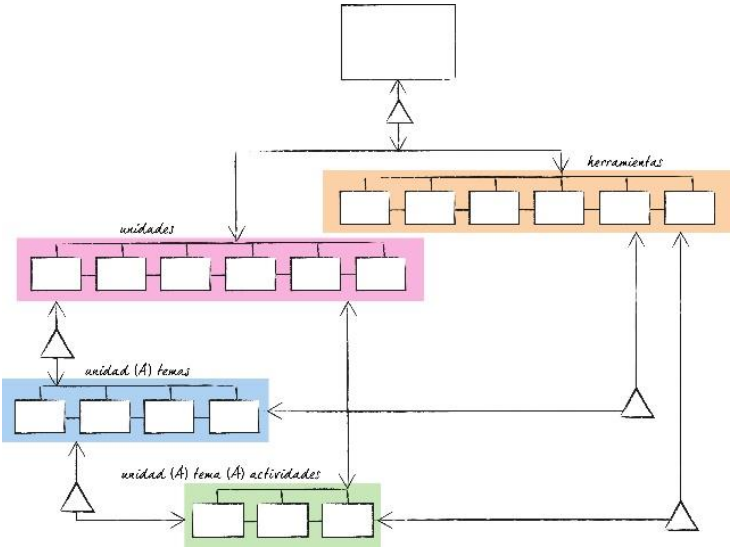

Figura 2. Mapa de sitio, se ejemplifica el mapa que considera una unidad

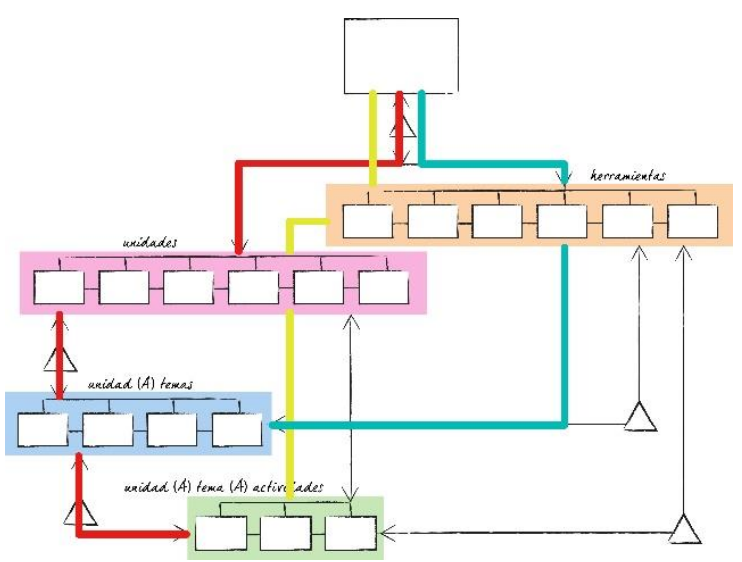

Figura 3. Flujo de navegación

La experiencia de usuario se conforma por todos aquellos elementos que intervienen en la satisfacción global de uso de un sitio y cuyo resultado es una percepción negativa o positiva del mismo. Peter Morville (2004), habla de siete aspectos que es importante considerar para que la experiencia del usuario sea positiva. Estos aspectos son: que el sitio sea útil, usable, deseable, localizable, accesible creíble, y valioso; esto muestra un amplio panorama de uso, ayuda a definir y balancear prioridades, y propone un equilibro basado en los objetivos del proyecto y las necesidades del usuario.

\section{2a. fase: Estructuración de la información.}

Una vez elaborado el mapa del sitio, se comienza el trabajo de estructuración y bocetaje o wireframe. El wireframe es una guía visual donde se define el área asignada a cada uno de los elementos que conformarán la interfaz gráfica, incluyendo la ubicación de los sistemas de navegación del sitio. Es importante utilizar técnicas de composición para resolver exitosamente esta segunda fase. La disposición de los elementos debe ser consistente, fácil de reconocer y de aprender, además debe jerarquizar la información que se presente en la interfaz.

La inserción de esta fase es el resultado de la consideración de aspectos especializados que se describen en la primera fase, como se muestra en la Figura 4 el wireframe hace tangible la conceptualización del sitio, conecta el mapa de sitio que se generó en la primera fase con la solución gráfica que se trabajará en la tercera fase de desarrollo.

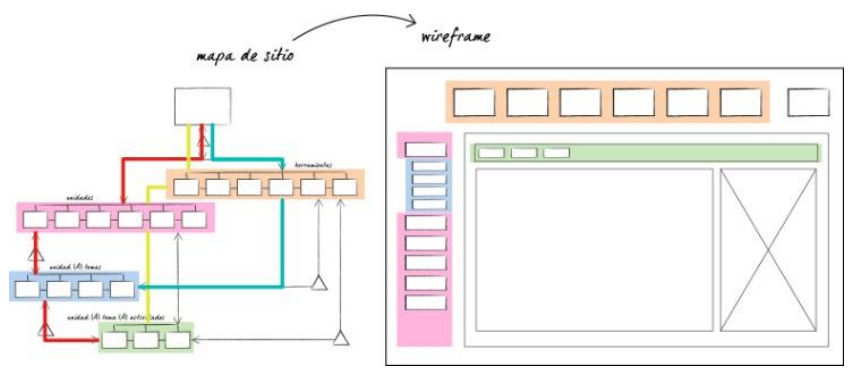

Figura 4. Materialización de la conceptualización del sitio 
3a. fase: Estética y comunicación visual

Se podría decir que este momento del desarrollo siempre se ha mantenido, aunque es importante mencionar que el trabajo se ha especializado y enriquecido con el tiempo y la experiencia. Centrar el trabajo en las necesidades de los usuarios nos brinda la pauta de trabajo para concretar la planificación del proyecto donde se define el problema, el público meta y las estrategias para llegar a la solución deseada.

En esta fase se trabaja con el aspecto visual de la interfaz y se define el estilo y diseño textual. En la CED la propuesta del diseño de interfaz gráfica se trabaja poniendo especial atención en la cualidad subjetiva y emotiva del gusto; según Donald Norman (2003) y el diseño emocional, cuando un producto es agradable a la vista y por lo tanto placentero lo que el usuario experimenta es un sentimiento positivo que lo vuelve más creativo y le facilita la solución de problemas, además estas emociones modifican la capacidad de atención, memorización y rendimiento del usuario.

Una vez definida la solución de la interfaz gráfica se debe trabajar en el diseño textual haciendo una jerarquización visual del contenido, para ello es importante considerar factores como la legibilidad y el $F$ pattern y así ayudar al usuario a realizar un escaneo más ágil sin perderla atención en el contenido. En esta tercera fase es importante utilizar estrategias de comunicación visual para que el resultado satisfaga las necesidades planteadas en el problema de diseño de cada uno de los proyectos.

4a. fase: Integración de contenido

Finalizadas las fases anteriores se puede considerar una 4ta fase, la de construcción e integración, y es en esta fase dónde se construya el layout y se realiza la integración del contenido mediante lenguajes de programación. Los lenguajes que se utilizan para la construcción y la integración, sin tomar en cuenta funcionalidades avanzadas como bases de datos, etc. son dos: HTML5 y CSS3. Al ser la tecnología inherente al desarrollo de estos proyectos, el estándar de construcción que se sigue en la CED es marcar el contenido y el prototipo con código HTML y utilizar CSS para controlar el aspecto visual, de esta forma se genera un sitio web estable en los diferentes navegadores y sistemas operativos. Esta separación facilita la actualización y ahorra tiempo, dinero y esfuerzo, ayuda al posicionamiento de la página, mejora la accesibilidad, proporciona a los usuarios flexibilidad al acceder al contenido, y permite que se presente el contenido en diferentes versiones: por ejemplo para verlo en pantalla, para imprimir, para proyección, etc.

Con el paso de los años en la CED el diseñador ha ido ganado terreno en la realización de esta fase, al inicio sólo definía el estilo visual y se quedaba al margen de la construcción del entorno virtual, ahora es capaz de participar en la construcción del prototipo y la integración del contenido lo que le permite tener el control sobre la presentación del contenido para proponer soluciones eficaces, eficientes y agradables que toman en cuenta elementos cognitivos y emocionales, aplicando principios de la Psicología Cognitiva a través del diseño. La psicología cognitiva estudia cómo percibimos las formas y los colores, y por qué en un ambiente lleno de estímulos somos capaces de discriminar y centrar nuestra atención en uno solo. Estas consideraciones son importantes para explicar cómo los seres humanos interactuamos con las máquinas.

En las siguientes figuras ejemplifica la evolución del trabajo del diseño de interfaz gráfica de usuario en la CED contrastando dos soluciones gráficas, una (Figura 6) cuyo trabajo se centró en el usuario y consideró todos los elementos que se han ido sumando con el paso del tiempo y otra (Figura 5) que estaba limitada al look \& feel del sitio y sólo consideró al responsable del proyecto y los programadores para el trabajo.

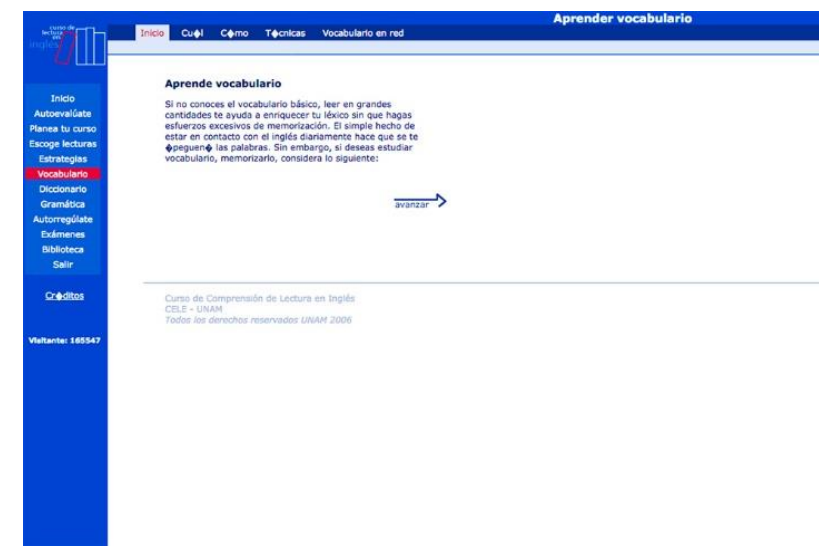

Figura 5. Sitio desarrollado en 2006

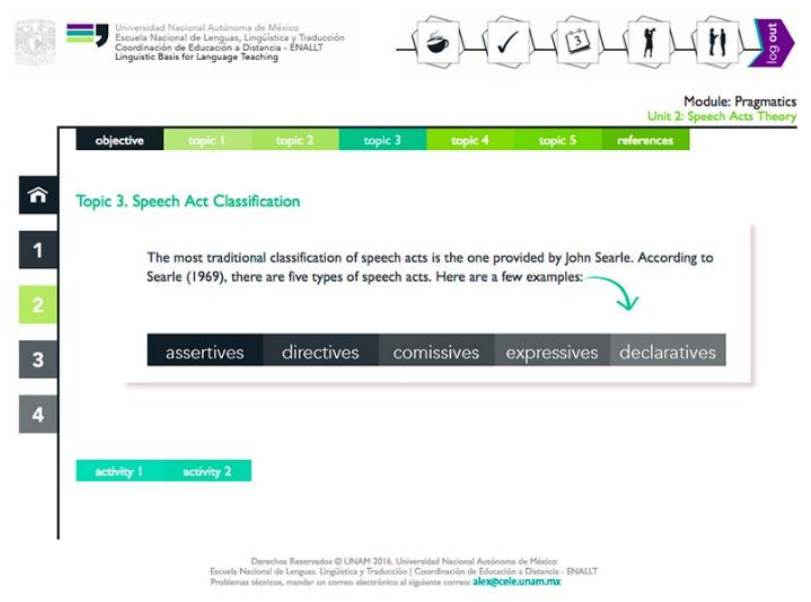

Figura 6. Sitio desarrollado en 2015

A simple vista se nota la diferencia de las figuras anteriores (Figuras 5 y 6), "una imagen dice más que mil palabras". Observando estas soluciones gráficas, es importante reconocer la necesidad de que el diseñador y comunicador visual participe activamente desde la 1a. fase, Joan Costa define al diseño gráfico como una actividad mediadora y al diseñador y comunicador visual como el responsable de la puesta en forma visual del mundo, el diseño gráfico no solo facilita la realización de funciones o acciones, transmite información. Es por eso que, 
como se distingue en la Figura 6, desde el marco conceptual del proyecto se deben utilizar elementos disciplinares y metodológicos propios del diseño y la comunicación visual, para que el resultado sea satisfactorio y sobre todo exitoso.

La Figura 5 muestra una solución que no consideró el trabajo de la primera y segunda fase que se describieron previamente, es una solución dónde no se organiza, clasifica y describe claramente el contenido del sitio web lo que resulta en una falta de jerarquía establecida con sistemas de navegación poco claros y la solución gráfica poco atractiva. En cambio, la Figura 6 muestra cómo ha evolucionado el trabajo de desarrollo, es una propuesta gráfica mucho más arriesgada que toma en cuenta estrategias de comunicación visual, la arquitectura de la información arroja sistemas de navegación claros, y se jerarquiza el contenido lo que facilita al usuario escanear el sitio y encontrar lo que busca.

El estudio de una lengua extranjera a diferencia de otras disciplinas requiere de un aprendizaje en comunidad por lo que el diseñador gráfico debe resolver una interfaz gráfica amigable que guíe en todo momento al alumno, que se sienta acompañado facilitando el trabajo en equipo y le permita encontrar lo que busca en cualquier momento para lograr trabajar diferentes habilidades.

La evolución en el área de ingeniería y programación en la etapa de desarrollo

\section{1a. fase: Producción e integración}

En la etapa de desarrollo se encuentra la fase de producción e integración donde se hace evidente la participación de los expertos en tecnología, es aquí donde estos profesionales se encargan de implementar las soluciones tecnológicas que han sido identificadas para que el proyecto se concrete.

Internamente los expertos en tecnología de la CED seguimos una metodología de desarrollo software que permite estructurar y controlar las tareas enfocadas a generar las aplicaciones tecnológica, entendidas como el conjunto de objetos digitales de aprendizaje, plataformas de cursos y sitios web requeridos por los proyectos. La elección del tipo de metodología depende de la naturaleza del proyecto, sin embargo, sin importar cuál sea la elección, en la CED tenemos identificadas como imprescindibles las siguientes etapas de desarrollo: análisis de requerimientos, implementación de aplicaciones, evaluación y pruebas, mantenimiento y actualización.

En el análisis de requerimientos, además del punto de vista técnico, se deben considerar los puntos de vista del resto de las disciplinas involucradas. Para tener estas perspectivas los expertos en tecnología deben realizar el análisis de necesidades desde las etapas iniciales del desarrollo para conocer y entender aspectos como la justificación del proyecto, los objetivos que persigue y las estrategias que lo sustenta; a su vez deben establecer una comunicación de calidad con los responsables de la asesoría pedagógica concertar acuerdos sobre cómo la tecnología puede potenciar el modelo pedagógico y las estrategias didácticas, y finalmente deben establecer consensos con los diseñadores para definir la concordancia entre el diseño de la interfaz y la implementación de funciones de las aplicaciones digitales.

Este intercambio de ideas y retroalimentación con el resto de colaboradores debe proporcionarle información suficiente que les permita a los expertos en tecnología tomar desiciones adecuadas para llevar a cabo la siguiente fase de implementación de aplicaciónes.

Una de las cuestiones más importantes sobre la implementación de aplicaciones es la búsqueda de la calidad de los desarrollos debido a que la calidad tiene repercusiones directas en la experiencia de uso de nuestros usuarios finales, y a su vez injerencia en las posibilidades de lograr los objetivos de enseñanza-aprendizaje. Existen diferentes normas o estandares que establecen marcos de referencia para asegurar la calidad de las aplicaciones, en la CED se ha elegido la norma ISO $25000 \mathrm{y}$ su apartado IEC 25010 como referencia de la calidad del software, esta norma establece un grupo de características deseables para cualquier aplicación de software mediante las cuales es posible dar una valoración de calidad en función de la experiencia de los usuarios con el uso de las aplicaciones. A continuación se definen las características de calidad de la norma ISO 25000:

Funcionalidad.- se refiere a que la aplicación educativa funcione como se espera que lo haga, para lo cuál se requiere recoger las opiniones de todos los involucrados en el desarrollo y establecer consensos para garantizar funcionalidad desde el punto de vista de contenidos, de la pedagogía, del diseño y de la tecnológia.

Confiabilidad.- se refiere a la certeza que pueden tener los usuarios de que las aplicaciones funcionen de la misma forma cada vez que son utilizadas.

Usabilidad.- se refiere a la capacidad de las aplicaciones para ser entendidas, aprendidas, usadas y atractivas para el usuario, en este sentido cuanto más sencillas e intuitivas logremos que sean nuestras aplicaciones menor será el esfuerzo que los usuarios tendrán que realizar para utilizarlas. Eficiencia.- se refiere al desempeño optimo de las aplicaciones en relación con los recursos necesarios para lograr dicho desempeño y una vez más vemos la necesidad de considerar el contexto tecnológico de los usuarios, específicamente el tipo de hardware y software al que tienen acceso para procurar que las aplicaciones que desarrollamos no requieran más recursos de los que este contexto ofrece, los usuarios deben experimentar el mejor desempeño sin tener que adquirir para ello nuevos recursos tecnológicos que les impliquen algun tipo de gasto.

Compatibilidad.- se refiere a la capacidad de las aplicaciones de llevar a cabo sus funciones en uno o más entornos digitales (dispositivos, sistemas operativos, navegadores, etc).

Seguridad.- se refiere a la capacidad de protección de la información y los datos de manera que personas o sistemas no autorizados no puedan tener acceso a los mismos. 
2a. fase: Evaluación del proyecto

Una vez que se concluye la producción e integración de un producto educativo (curso, sitio o material) se pasa a la siguiente fase dentro del desarrollo proyectos: evaluación, ajustes y entrega. En esta fase la evaluación se realiza de forma interna y externa tanto en el aspecto de funcionalidad como en el del contenido académico. En primera instancia la revisión interna hecha por otro miembro de la CED, de preferencia ajeno al proyecto a evaluar, nos arroja una lista de observaciones sobre los aspectos técnicos que es importante reconsiderar antes de liberar el producto; comúnmente estas observaciones son de usabilidad y en menor medida de funcionalidad; la evaluación externa versa principalmente sobre el contenido y al final la decisión de cambiar algo en ese sentido recae en el responsable académico del proyecto.

En el aspecto técnico, se realizan todos los ajustes que se consideren necesarios antes de liberar una versión beta que pueda ser ya un instrumento de pilotaje para los profesores e incluso después de esto se vuelven a realizar ajustes que mejoren la experiencia de usuario para futuras reediciones de los productos. No obstante, la evaluación del proyecto no termina ahí, esta tarea se realiza de manera continua y acompaña al proceso de la etapa del post-desarrollo donde se realizan las actualizaciones continuas de todos nuestros desarrollos.

En este sentido, en la CED se procura estar al día con el surgimiento de las nuevas tecnologías, evaluando la pertinencia de realizar cambios en cada una de las aplicaciones en funcionamiento en pro de mejorar la portabilidad y la eficiencia y con ello brindar productos educativos de calidad a los profesores y alumnos. Para ello, un tipo de evaluación que se realiza en los proyectos es el monitoreo de la experiencia de uso mediante la retroalimentación que recibimos de los alumnos a través de un cuestionario de evaluación que se aplica una vez que finaliza la impartición de un curso o módulo de diplomado.

Cabe destacar que el instrumento que actualmente usamos para la evaluación de los proyectos ha ido cambiando conforme a las necesidades de la ENALLT, inició como un instrumento particular para evaluar al diplomado ALAD y posteriormente se modificó para generalizarse y sistematizarse en un cuestionario de reactivos cerrados tipo Likert, con 39 ítems y estructurada en siete apartados: información general; estructura; estrategias instruccionales; recursos, materiales y actividades de aprendizaje; diseño de interfaz gráfica; programación y sistemas y el desempeño de los asesores. Estos apartados tienen relación con cuatro de las dimensiones propuestas por Badrul Khan (2005): pedagógica, tecnológica, diseño de interfaz y soporte. Este instrumento nos ofrece la ventaja de generar reportes y estadísticas de una manera más sencilla ya sea para tener un panorama general o un análisis por apartado; también nos da la oportunidad de conocer la opinión sobre todo el producto porque al final de los reactivos se incorporó un espacio para que los participantes pudieran expresar su opinión personal libremente (Rodríguez, 2016).
Como expertos en tecnología, nuestro interés en los resultados se enfoca en investigar cómo se sintieron al navegar en las páginas, en la usabilidad y en la funcionalidad de las aplicaciones, si se cumplió o no con esa serie de características que ya hemos mencionado tanto en diseño gráfico como en programación y uso de tecnología.

De manera general, el área de minería de datos web tiene como propósito estratégico la predicción del comportamiento de los usuarios del sitio, la comparación entre el uso esperado y real del sitio web y el ajuste del sitio web con respecto a los intereses de sus usuarios; mientras que el análisis web se centra en la mejora de la comunicación web mediante métricas y patrones de uso (Dragos, 2009). En la CED, el uso de la herramienta Google Analytics nos ha permitido obtener datos demográficos y datos sobre el uso de tecnología como los tipos de dispositivos, sistemas operativos, navegadores y resoluciones de pantalla que para el área tecnológica son de gran relevancia porque nos da un panorama muy completo del comportamiento de nuestra población meta y en base a ello se sustenta la toma de decisiones sobre el uso o desuso de herramientas tecnológicas, la adaptabilidad que se requieren en el diseño gráfico y el énfasis que debemos dar en a las pruebas de funcionalidad e integración para que sean útiles para la mayoría.

Los datos derivados de la encuesta de evaluación y del análisis web por si mismos no constituyen un indicador del aprendizaje del contenido de nuestros cursos y sitios en línea, pero sí creemos que puede ser el inicio para revisar lo que se está investigando sobre el uso de la minería de datos para la mejora de contenidos y actividades de aprendizaje basados en web (Friesen, 2013). Lo interesante de esta recopilación de datos es el uso que se haga de ellos para entender el comportamiento del estudiante y los patrones de aprendizaje mientras se trabaja en un entorno de aprendizaje en línea y que esto impacte en la toma de decisiones más allá de los aspectos técnicos del proceso de desarrollo.

Todo este proceso se ha ido mejorando en estos años no solo por la experiencia adquirida sino también por la diversidad de los proyectos que hemos tenido la oportunidad de trabajar en la CED, en consecuencia, es un hecho que continuará modificándose y adaptándose en base a los nuevos retos que se plantee la ENALLT.

La evolución en el área de ingeniería y programación en la etapa de post-desarrollo.

En la CED, la etapa de post-desarrollo empieza una vez que se entrega el proyecto y los usuarios hacen uso de la aplicación; en ese momento, el trabajo del área de ingeniería cambia, enfocándose en las fases de administración, mantenimiento y actualización. En la CED, estas fases se desarrollan según sean requeridas y con la periodicidad que cada proyecto impone.

La administración técnica de las aplicaciones consiste en realizar las tareas necesarias para que los sistemas sean utilizados por los usuarios, es decir: revisión de funcionalidad, gestión de usuarios, configuración de plataforma y actividades, soporte técnico a usuarios y creación de respaldos de sistemas. 
La fase de mantenimiento implica realizar modificaciones a los sistemas y aplicaciones, las cuales se pueden clasificar de acuerdo a la razón por la que son necesarias en los siguientes tipos: de corrección cuando los cambios se deben a la solución de problemas detectados durante su funcionamiento, de adaptación cuando responden a los cambios y nuevas demandas de su entorno, y de mejora cuando surge la necesidad de añadir funciones adicionales que van más allá de sus requisitos funcionales originales.

Respecto a la actualización, la naturaleza de cambio y evolución de la tecnología obliga a los expertos en tecnología a mantenerse al día para identificar áreas de oportunidad, desde el punto de vista técnico, que contribuyan a un mejor logro de los objetivos de los proyectos de enseñanza de lenguas digitales y sean congruentes con las necesidades actuales.

En este sentido, la CED inició con un proyecto de educación continua dirigido a profesores de lenguas, llamado “Actualización en Lingüística Aplicada a Distancia” (ALAD), para lo cual se resolvió utilizar una plataforma para gestión de cursos desarrollado ad-hoc por personal del entonces CELE que atendiera las necesidades específicas de ese proyecto.

Sin embargo, ALAD se creó en un contexto donde la demanda de oferta educativa en línea de la ENALLT apenas comenzaba a manifestarse, la consolidación de la CED genera la llegada de múltiples nuevos proyectos que debían ser desarrollados con prontitud por lo que se presentó la disyuntiva de generar una plataforma para cada uno o considerar otras alternativas. Por razones como el número de usuarios potenciales, la impartición simultanea de cursos y la posibilidad de centralizar la administración técnica, la decisión se inclinó por utilizar un Learning Management System entendido como la aplicación que provee la infraestructura de ambiente multiusuario donde desarrolladores, diseñadores instruccionales y expertos en contenidos pueden crear, almacenar, reutilizar, gestionar y proveer contenidos de aprendizaje digitales. El LMS elegido fue Moodle por tratarse de un sistema con licencia de uso gratuita y de código abierto. Este sistema ofrece las capacidades necesarias para soportar múltiples cursos, cantidades significativas de usuarios así como la impartición simultánea de un mismo curso. En cuanto a la administración, amplía las capacidades aunque también es cierto que este excedente no siempre es requerido. Por otro lado al tratarse de un sistema creado por terceros el conocimiento y entendimiento de la infraestructura de programación y base de datos es una tarea significativa por lo que los requerimientos de cambios e incrementos de funcionalidades modificando al propio sistema son tareas más complejas y que requieren mayor tiempo comparado con las plataformas de desarrollo propio, pero esto se compensa con la prontitud con la que el sistema puede ser instalado y configurado para su uso y con la existencia de los plugins para este tipo de sistemas que agregan funcionalidades de forma transparente.

Actualmente, en la CED se mantiene vigente tanto la plataforma ALAD como el LMS Moodle, este último soporta alrededor de
50 ambientes digitales de enseñanza aprendizaje entre cursos y módulos de diplomados.

\section{Conclusiones}

El uso de Internet como medio y recurso ofrece muchas ventajas debido a su capacidad para acercar y comunicar. Conforme avanza la tecnología se integran nuevos recursos que mejoran las posibilidades de mostrar el contenido, aumentar la interacción, el trabajo colaborativo y el autoaprendizaje. Actualmente la necesidad comunicativa se satisface integrando varios elementos; de la misma forma la comunicación se ha modificado, la manera como adquirimos los conocimientos también.

En este sentido, el equipo de desarrollo tecnológico de la CED ENALLT realiza su trabajo de manera multidisciplinaria centrándose en el estudiante, para que la experiencia de éste sea positiva y alcance los objetivos planteados considerando la interculturalidad que conlleva la enseñanza/aprendizaje de lenguas. Asimismo, la CED está muy interesada en desarrollar entornos virtuales que sean accesibles para todos los estudiantes y por ahora pretende incluir en los nuevos proyectos los lineamientos mínimos de accesibilidad web que incluye en principio a la comunidad con debilidad visual.

\section{REFERENCIAS}

Cañas, J.J. \& Waerns, Y. (2001). Ergonomía Cognitiva: Aspectos Psicológicos de la Interacción de las Personas con la Tecnología de la Información. España, Editorial Médica Panamerican S.A.

Costa Joan, (2014) Diseño de comunicación visual: el nuevo paradigma. En Gráfica, documentos de diseño gráfico (pp. 89 - 107). ISSN (en línea) 2014-9298

Dragos, S., Dragos, R. (2009). WATEC: a Web Analytics Tool for Educational

Content. Proceedings of the International Conference on Knowledge Engineering, Principles and Techniques, KEPT2009, Cluj-Napoca (Romania), July 2-4, 2009. (pp. 25-31)

E-Learning Framework for Massive Open Online Courses by Badrul H. Khan. Recuperado de: http://badrulkhan.com/

Friesen, N. (2013). Learning Analytics: Readiness and Rewards / L'analyse de l'apprentissage : état de préparation et récompenses. Canadian Journal of Learning and Technology / La revue canadienne de l'apprentissage et de la technologie, 39(4). doi: http://dx.doi.org/10.21432/T2J01B

Khan, B. (2005). Managing E-Learning Strategies. USA: INFOSCI

Mishra, P., \& Koehler, M. J. (2006). Technological Pedagogical Content Knowledge: A framework for teacher knowledge. Teachers College Record, 108(6), 1017-1054. Recuperado de http://one2oneheights.pbworks.com/f/MISHRA_PUNYA.pdf 
Pérez-Montero, Mario (2009). Arquitectura de la información en entornos web. Cenero, Gijón: TREA.

Rodríguez, A., Baca, E. (Panelistas y Coordinadores Académicos). (2016, noviembre 1). Antes de empezar, hay que planear (programa de TV). J. San Pablo (Productor), Serie Vamos por partes: desarrollo de cursos de lengua en línea. Ciudad de México, MX: TV UNAM.

Ronda León, R., La diagramación en la arquitectura de la información. Recuprado de: www.nosolousabilidad.com

Rodríguez, H. (2012, 24 de febrero). WIAD 2012 - Ergonomía cognitiva aplicada al diseño de arquitecturas de información. [video]. Recuperado de: https://www.youtube.com/watch?v=ra1Yc2zdQwQ

Semantic Studios by Peter Morville. Michigan, EU. Recuperado de http://semanticstudios.com/

Wood, D. (2015). Diseño de Interfaces. Introducción a la comunicación visual en el diseño de interfaces de usuario. Barcelona, Parramón Paidotribo.

(2017) ISO/IEC 25010. ISO 25000. Recuperado de http://iso25000.com/index.php/normas-iso-25000/iso-25010. 